\title{
Laser Soldered Eutectic Die-Bonding Processes in LED Packaging
}

\author{
Te-Ching Hsiao, Ah-Der Lin, ${ }^{1 *}$ and Wei-Yi Chan ${ }^{2}$ \\ Department of Mechanical Engineering, \\ National Kaohsiung University of Applied Science, Kaohsiung 80778, Taiwan \\ ${ }^{1}$ Department of Mechanical Engineering, Cheng Shiu University, Kaohsiung 83347, Taiwan \\ ${ }^{2}$ Department of Mechanical and Electromechanical Engineering, \\ National Sun Yat-sen University, Kaohsiung 80424, Taiwan
}

(Received August 31, 2015; accepted March 23, 2016)

Keywords: die-bonding, eutectic, laser, thermal stress, residual stress

This work investigated the effect of laser power patterns on temperature and thermal stress distributions in the light emitting diode (LED) die-bonding process. The finite element software package MSC.Marc was employed to simulate the laser soldering process. Thermal-elastic-plastic models of the solid elements were used. Temperature dependent material properties were applied to characterize the temperature variation effect that occurred during die-bonding. This research simulated and studied temperature and thermal stress distributions during the die-bonding process, as well as the distributions of residual stress induced in the die-bonding process and the effects of different laser soldering parameters on die-bonding. The results show that thermal stress and residual stress are affected by process temperature quality, and that residual stress in the tin alloy increases as the trough depth increases.

\section{Introduction}

Energy issues have attracted an increasing amount of attention in recent years; for example, the light emitting diode (LED) has become the perfect choice to upgrade the efficiency of energy use as semiconductor manufacturing technology continues to progress. The LED die-bonding process can be divided roughly into the epitaxy process, the die process, and packaging and testing, where packaging and testing refer to placing bonding materials on electric circuits and then pasting crystal grains directly on the circuits. Then through die-bonding thermal treatment, those crystal grains can be fixed onto a substrate, thereby completing the process. Wire bonding is used depending on the packaging mode as current channels for the anodes and cathodes of the crystal grains.

Most traditional die-bonding processes are completed by using silver pastes; however, as the power was increased, problems such as high packaging thermal resistance values, degradation, and poor bonding strength appeared. To improve LED quality, eutectic bonding technology was developed, which applied eutectic metals to bond crystal grains directly onto a sub-mount. This process had better heat transfer ability than the silver colloid and also increased the operating power, and provided better bonding strength. To improve eutectic bonding and control accuracy, it introduced laser technology with high-energy density to create rapid and accurate localized heating.

*Corresponding author: e-mail: ahder.lin@gmail.com 
Lin adopted the localized heating mode in 1998 to heat gold and spread it into a silicon substrate, thus achieving eutectic bonding.(1,2) Wild et al. in 2001 then bonded glass and silicon substrates together using a laser. ${ }^{(3)}$ In 2004, Tao et al. packed micro-electromechanical systems (MEMS) onto a ceramic substrate also using a laser. ${ }^{(4,5)}$ In 2005, Tan et al. used a Nd:YAG laser soldered eutectic die-bonding technology and eutectic materials to bond onto a silicon substrate. ${ }^{(6,7)}$

Eutectic bonding technology involves changes in materials, which cause plastic deformation inside an alloy and trigger residual stresses within each material due to the coefficient of thermal expansion (CTE) mismatch. Under LED operations, the residual stresses are superimposed on operating thermal stresses, leading to fatigue and thus reducing reliability. Therefore, residual stress is the key point of eutectic bonding technology. In 2007, Teo et al. used many tin gold alloys before packaging to obtain advantages in alignment, mechanical properties, and performance. ${ }^{(8-12)}$ This study adopted the finite element method together with the simulated laser eutectic bonding process. As properties of materials change with temperature, thermal stresses and residual stresses produced during the process are discussed, and each material's temperature and stress changes associated with various process parameters are analyzed.

\section{Basic Principles and Theory}

\subsection{Heat transfer analysis theory}

Through heat transfer analysis, the heat transfer equation was derived from the first-order Fourier expansion formula, namely:

$$
q_{k}=A k \cdot \nabla T,
$$

where $q_{k}$ is the rate of heat flow, $A$ is the region of study, $k$ is thermal conductivity, and $T$ is temperature.

The system must obey the energy balance equation,

$$
\nabla(k \cdot \nabla T)+\dot{e}=\rho C_{p} \dot{T},
$$

where $\rho$ is material density, $C_{p}$ is specific heat, and $\dot{e}$ is the rate of heat generated per unit volume.

Based on Newton's cooling law, the boundary condition for heat transfer in the material is:

$$
\operatorname{Ah}\left(T-T_{\infty}\right)=q_{c},
$$

where $h$ is the natural convection coefficient, $T_{\infty}$ is the ambient temperature, and $q_{c}$ is the rate of heat flow.

\subsection{Stress analysis theory}

The stress-strain within the material was then calculated using the strain increment relation formula:

$$
d \varepsilon_{i j}=d \varepsilon_{i j}^{e}+d \varepsilon_{i j}^{p}+d \varepsilon_{i j}^{t h},
$$


where $d \varepsilon$ is the total strain increment, $d \varepsilon^{e}$ is the elastic strain increment, $d \varepsilon^{p}$ is the plastic strain increment, and $d \varepsilon^{\text {th }}$ is the thermal strain increment. Among these, thermal stress is generated from the temperature change, namely:

$$
d \varepsilon_{i j}^{\text {th }}=\alpha_{i} d T_{j}
$$

where $\alpha$ is the CTE, and $d T$ is the temperature change.

Based on Hooke's law, the relationship between stress and strain within the material is obtained:

$$
d \sigma_{i}=C_{i j}^{e} d \varepsilon_{j}^{e}-C_{i j}^{t h} d T_{j},
$$

where $\alpha$ is the elastic tensor, and $C^{\text {th }}$ is the CTE matrix. In consideration of the material status in a balanced condition, each point within the system must comply with the following equation:

$$
\sigma_{i j, j}+B_{i}=0,
$$

where $\sigma$ is the stress tensor, and $B$ is body force; however, without regard to the effect caused by the non-contact force, this term can be ignored.

The constitutive equation of the thermoplastic material, based on the von Mises yield criterion, its relevant associated flow rule, and the isotropic strain hardening rule, can be written as:

$$
f\left(\sigma_{i j}, \bar{\varepsilon}_{p}, T\right)=\frac{3}{2} S_{i j} S_{i j}-\sigma_{e}^{2}\left(\overline{\varepsilon_{p}}, T\right)=0,
$$

where $S$ is the derivative stress tensor, $\sigma_{e}$ is the effective stress, and $\bar{\varepsilon}_{p}$ is the effective plastic strain. Equation (6) can be rewritten as:

$$
d \sigma_{i}=C_{i j}^{e} d \varepsilon_{j}^{e}+C_{i j}^{p} d \varepsilon_{j}^{p}-C_{i j}^{t h} d T_{j},
$$

where $C^{p}$ is the plastic tensor.

\section{Establishing the Finite Element Model}

In this study, the laser eutectic die-bonding process during LED packaging was analyzed using the finite element package software MSC.Marc for numerical simulation.

\subsection{Structure and contact setup}

The structure consists of a die and bearing substrate with the substrate bonded with a bond pad in the eutectic mode, and then electroplated onto a silver bonding pad, thereby forming a new matching surface with the circuit of each crystal grain connected to create LED modules in matrix form, as shown in Fig. 1. To save calculation time and simplify the process, we studied only one or two modules within the matrix. Crystal grains used for the study were manufactured by CREE, DA1000, with the luminescent layer being $\mathrm{InGaN}$ and the substrate being $4 \mathrm{H}-\mathrm{SiC}$. A premetallized tin alloy on an epitaxial layer simplified the structure further to a monolayer of gallium nitride. 
As the eutectic tin alloy had the lowest melting point, we have divided it into $80 \%$ gold and $20 \%$ tin to have the metals in a mixed state as a eutectic material. The tin alloy also contained a cathode and an anode. The thickness of each layer from the bottom up was: substrate, $450 \mu \mathrm{m}$; copperplated layer, $30 \mu \mathrm{m}$; nickel-plated layer, $5 \mu \mathrm{m}$; silver-plated layer, $3 \mu \mathrm{m}$; tin alloy, $3 \mu \mathrm{m}$; InGaN, 10 $\mu \mathrm{m}$; and carbonized silicon substrate, $325 \mu \mathrm{m}$. A section of the matrix after simplification is shown in Fig. 2.

Owing to the significant difference in thickness of each part, eutectic mesh cutting was not available, and an adopted contact had to be set up. During the analysis, we assumed that there was no friction or thermal contact resistance between the two contacts; contact tolerance and tolerance deviation were both zero. Furthermore, to simulate different bonding modes during alloy fusion, we assumed that alloys and other contacts were all touching during laser heating. After the heat source was shut off, alloys and other contacts were turned into gel to investigate the alloy's fixed function when it was not bonding to other contacts and after it was solidified. Other contacts, whether exposed to the laser heat source or not, were all in a gel.

Constraints were set in a symmetrical direction in each layer. Moreover, to prevent carbonized silicon and gallium nitride from changing into free bodies due to segregation, a gram of equivalent weight was placed on the carbonized silicon in the form of increased pressure.

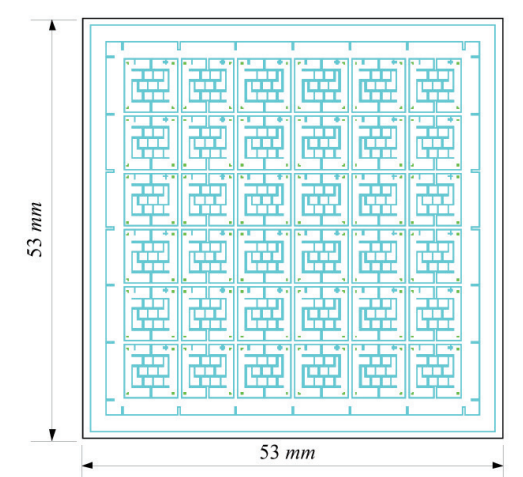

Fig. 1. (Color online) Matrix design of bonding pad.

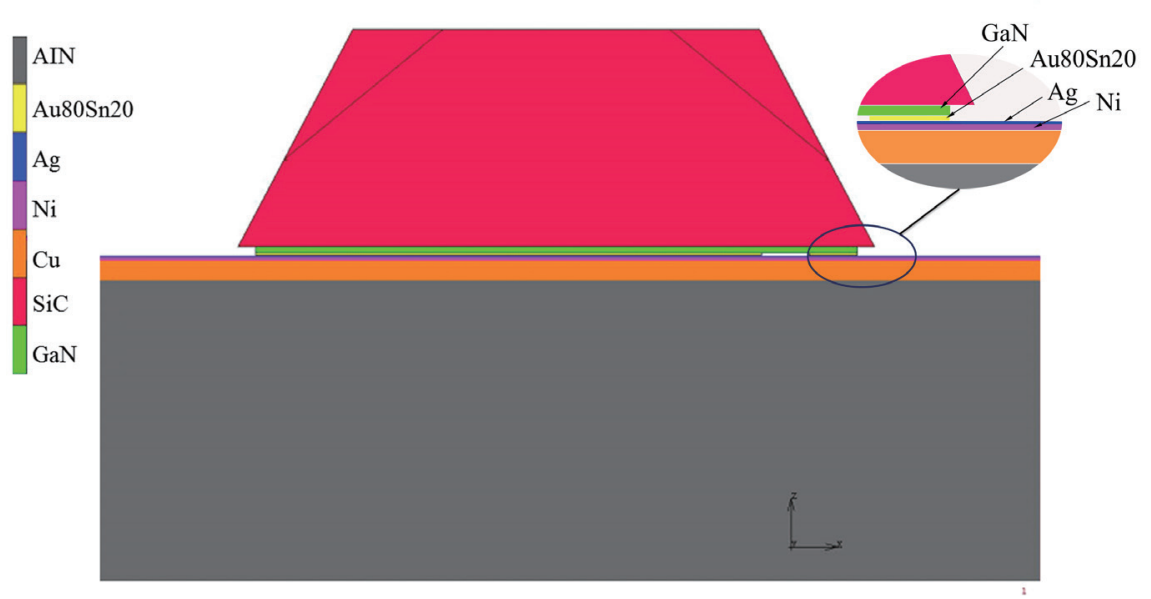

Fig. 2 (Color online) Section after simplification of matrix. 


\subsection{Mesh cutting and convergence analysis}

The models comprised 8-noded hexahedral low-order elements for mesh cutting, and trilinear interpolation was used to describe the physical distribution of quantities within each element. ${ }^{(13)}$ Owing to the extremely low percentage occupied by the region of interest in the entire region of material, the transient mode was adopted and convergence was used to analyze and confirm the reliability of the calculation. The proportion of the mesh's longest edge touching each contact was fixed at 2.5 times, which was uniformly distributed along the tin alloy from end to end. A total of seven models were set up and, taking the tin alloy's longest edge as the base, the models were 12 , $11,10,9,8$, and $7 \mu \mathrm{m}$ long, with a start-up time of $0.02 \mathrm{~s}$. Regions heated within $2.08 \mathrm{~s}$ were the focus of thermal stress, and the thermal stress distribution was selected within $1 \mathrm{~s}$ of heating for the purpose of discussion. Once removed from the heat source, the $5 \mathrm{~s}$ natural convection for cooling down was the second focus, for which the stable residual stress of $7.08 \mathrm{~s}$ was selected.

The numerical results illustrate that the residual stress had a stricter convergence requirement than the thermal stress, and that the mesh cutting of this part of the model was the key point. Figure 3 shows carbonized silicon's residual stress to 193433 elements when converged to $\pm 5 \%$, while gallium nitride converges to 146094 elements, and the tin alloy as well as the nickel bonding pad all converge to 193433 elements within $\pm 5 \%$. Here, a completely plastic model was used, and the copper and silver bonding pads were all maintained within the plastic region. Finally, the tin alloy models were selected for later use with the longest one being $8 \mu \mathrm{m}$ long, as shown in Fig. 4.

\section{Numerical Results and Discussions}

\subsection{Definitions}

A group of trial process parameters was set up to obtain the trends in temperature, thermal stress, and residual stress during the process, and then the destructive parts were determined for later discussion.

As for the substrate and its parts in contact with the bonding pad, because the aluminum nitride substrate has rigid mechanical properties, which always cause damage to materials, no special

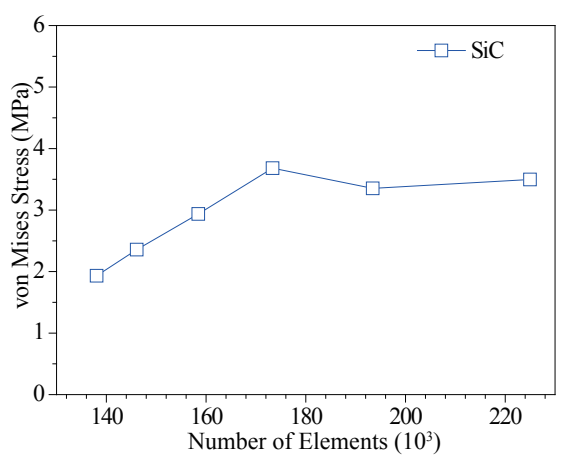

Fig. 3. (Color online) Residual stress convergence in carbonized silicon.

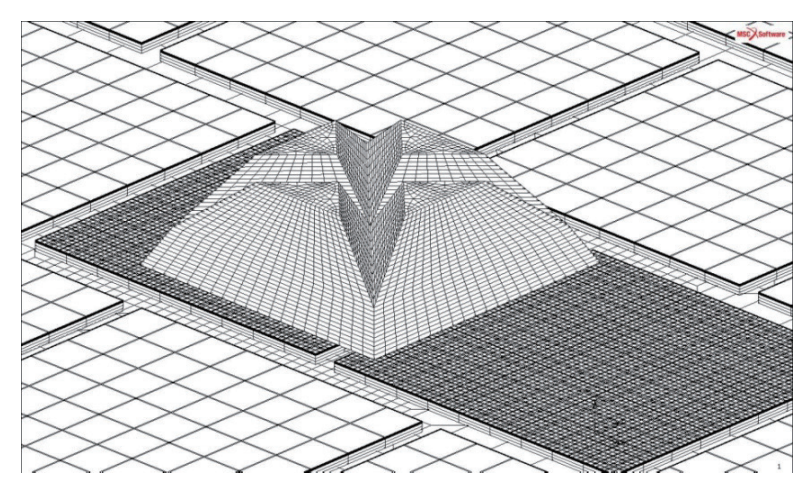

Fig. 4. Mesh cutting of overall region of interest. 
attention was given to it. The substrate was not the region of maximum tensile stress after removing the heat source. During the heating of the copper-nickel-silver bonding pad, and due to Gaussian distribution of the energy from the laser heat source, the temperature distribution is circular. Owing to the incompatibility between CTEs, stress was generated under these conditions.

The temperature distribution in the Au80Sn20 sample appeared as a concentric circle around the focal point of the laser. With respect to the flip chip LED (FCLED) structure, which caused the temperature distributions in both the anode and the cathode to decrease discontinuously, as shown in Fig. 5, the anode failed to reach its eutectic point temperature as the cathode was within large areas of fused material. To study the change in the tin alloy's temperature field, 12 temperature observation points, A-L, were selected and distributed as shown in Fig. 6. With respect to stress, we adopted Young's random temperature change modulus and yield strength and determined that Young's modulus under fusion is $200 \mathrm{MPa}$, while the yield strength is $0.5 \mathrm{MPa}$. Once cooled, the sample showed a residual stress of $100 \mathrm{MPa}$. The observation points for residual stress were set as AuSn_A and AuSn_B on the tin alloy, as shown in Fig. 7.

The difference between the highest temperature of the gallium nitride's epitaxial layer and that of tin alloy was less than $1{ }^{\circ} \mathrm{C}$; therefore, we did not need to fix any temperature observation
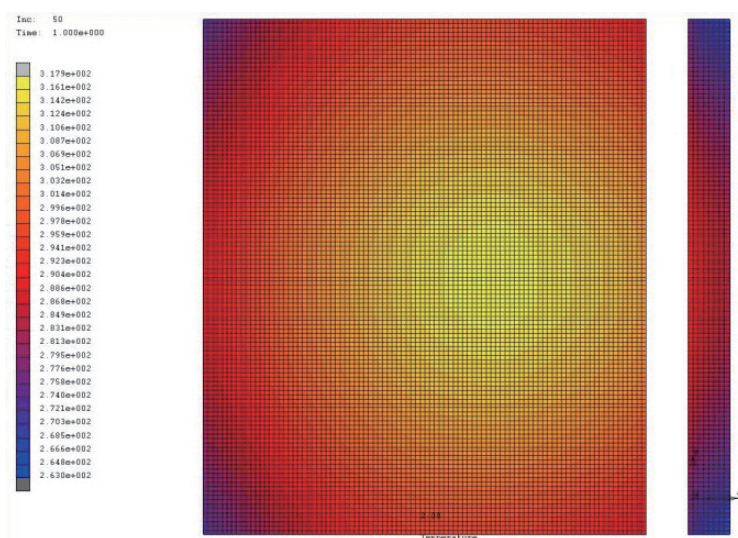

Fig. 5. (Color online) Temperature field distribution in the tin alloy after $1 \mathrm{~s}$ exposure to a laser.

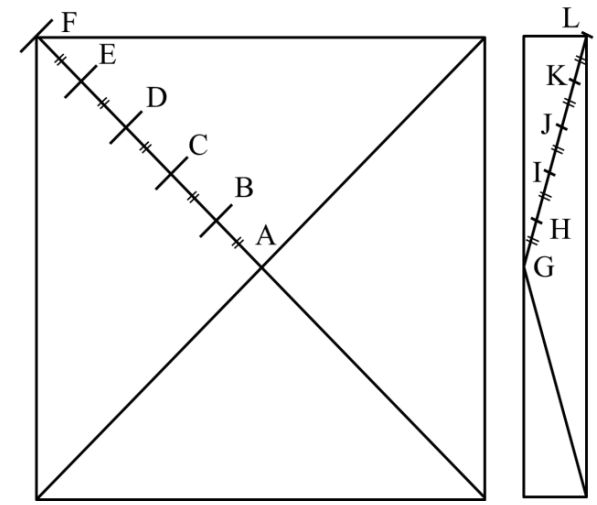

Fig. 6. The positions of the temperature observation points for each tin alloy layer.

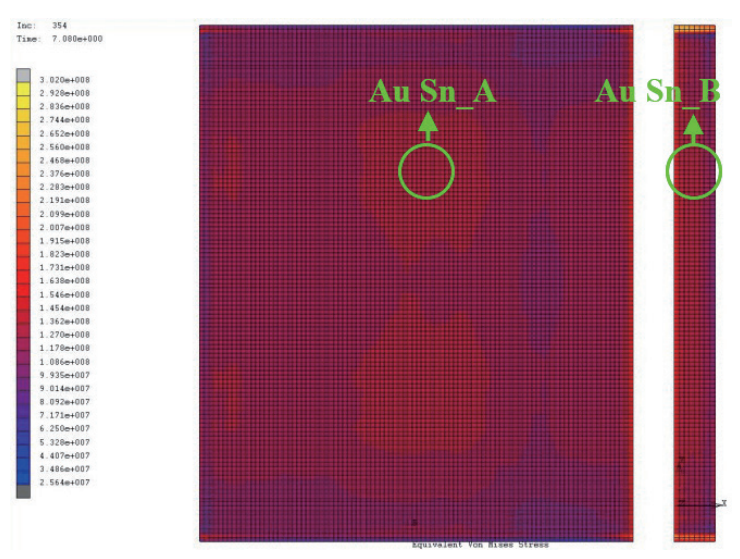

Fig. 7. (Color online) Observation points in the tin alloy. 
point. With respect to stress, the main maximum stress is negative, representing pressure stress status, which does not cause structural damage. Therefore, special concern for the change in the epitaxial layer's thermal stress is not needed. Once the sample cooled down, the main tensile stress on the epitaxial layer was distributed like that in the tin alloy layer and was divided between the cathode block and the anode block. Thus, it is inferred that the residual stress was triggered by the solidification of the tin alloy. The average von Mises stress was about $35 \mathrm{MPa}$, and residual stress observation points GaN_A and GaN_B were each taken from the cathode block and the anode block, as shown in Fig. 8.

Lastly, because the thickness of the epitaxial layer was only $10 \mu \mathrm{m}$, the temperature distribution of the carbonized silicon substrate was very similar to that of gallium nitride. The thermal stresses in the carbonized silicon substrate during laser heating caused an alternation between tension and compression. Here we determined Focus SiC_A, as shown in Fig. 9, as the maximum tensile stress point within the entire section, which was formed because of the unmatched expansion coefficients between the cooling and solidifying tin alloy, the epitaxial layer, and the carbonized silicon substrate once cooled. To see whether carbonized silicon would separate from the bottom, SiC_B was chosen as the second observation point, as shown in Fig. 10.
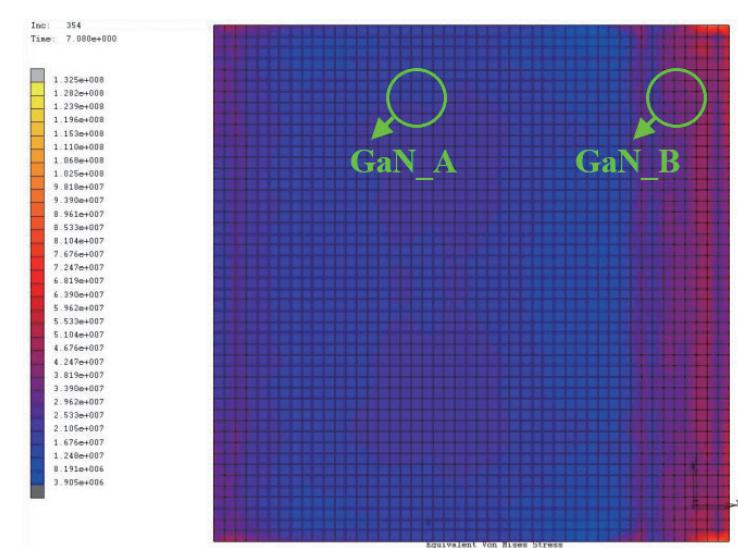

Fig. 8. (Color online) Distribution of observation points for epitaxial layer's residual stress.
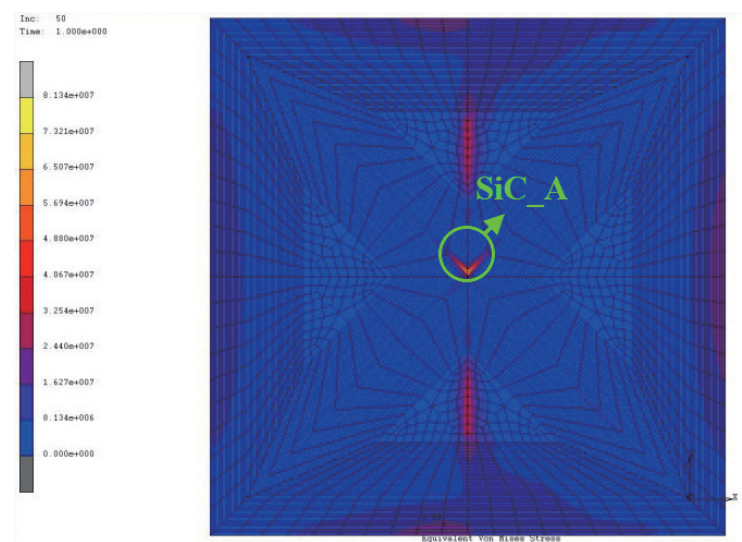

Fig. 9. (Color online) Focus of carbonized silicon substrate thermal stress.
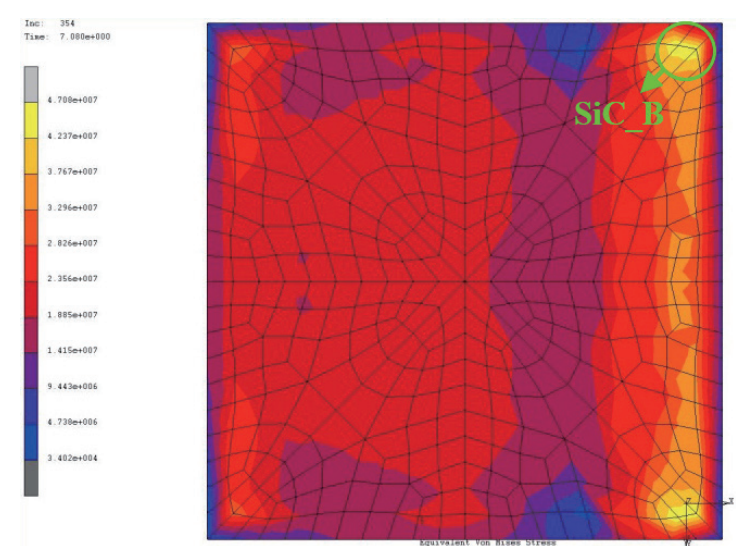

Fig. 10. (Color online) Residual stress focus of carbonized silicon substrate. 


\subsection{Parameter analysis}

\subsubsection{Laser deviation}

To solve the problem of uneven temperature field distribution in the cathode and the anode, deviation analysis was carried out in the vertical clearance direction. The $x$-bright cross-point was taken as the center; movement near clearance as the positive deviation; and the movement away from clearance as the negative deviation. During the study, we discussed whether Point E and Point $\mathrm{K}$ could be $90 \%$ bonding points and searched for the uniformity of anode and cathode by noting the temperature difference between these two points as the basis. The temperature difference change as the laser deviated, as shown in Fig. 11. When the positive deviation occurred from 0.05 to 0.1 $\mathrm{mm}$, the laser deviation showed zero temperature difference. According to distance, temperature difference, and linear interpolation, it is inferred that when the positive deviation was $0.08 \mathrm{~mm}$, the temperature difference between these two points was the smallest, which means the most even laser deviation distance. Figure 12 shows how the substrate thermal stress changed as the laser deviated, the trend of which also reversed at $\pm 0.2 \mathrm{~mm}$. The thermal stress in this region presented neither a significant change nor the risk of breaking down the substrate. Figure 13 shows each material's residual stress under such a deviation.

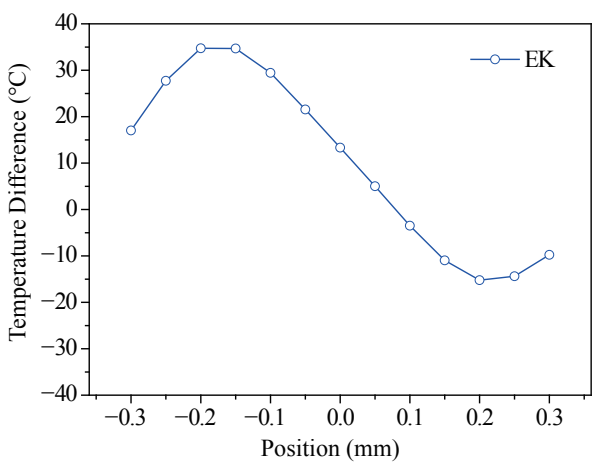

Fig. 11. (Color online) Temperature difference between Point $\mathrm{E}$ and Point $\mathrm{K}$ as a function of laser deviation.

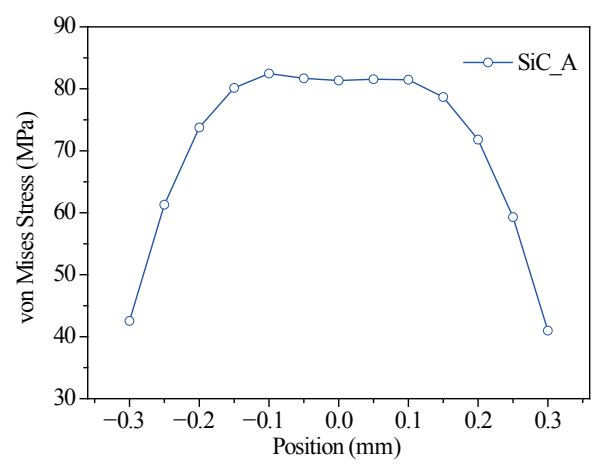

Fig. 12. (Color online) Thermal stress at Point SiC_A as a function of substrate thickness.

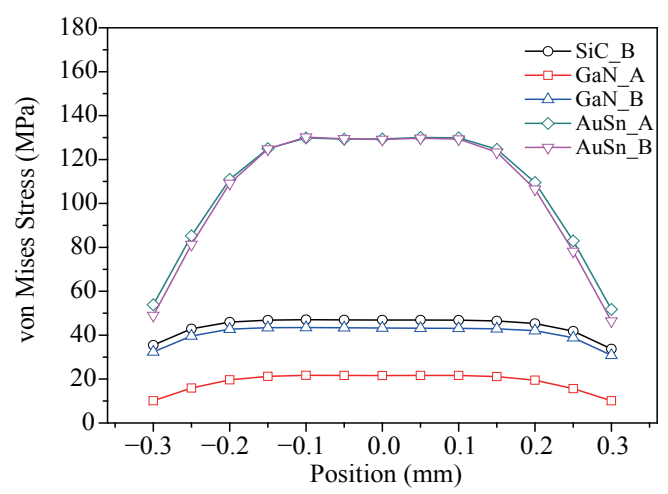

Fig. 13. (Color online) Change in residual stress at the observation point as a function of laser deviation 


\subsubsection{Substrate thinning}

The bearing substrate not only provides an electric metal layer attached to components, but also offers certain mechanical protection for the overall packaging, which is more important. Although a thick bearing substrate can afford the strongest mechanical protection, it also increases the packaging's thermal resistance. However, current packaging requires a lower thermal resistance with a thinner substrate. This section discusses the substrate thinning effect on the laser diebonding process, which is divided into five groups of thicknesses: the most commonly seen, 0.45 and $0.55 \mathrm{~mm}$; and three thinner groups, $0.35,0.25$, and $0.15 \mathrm{~mm}$.

Each temperature point of the tin alloy after $1 \mathrm{~s}$ of heating is shown in Fig. 14, and different substrates have considerably different effects on tin alloy temperature change for the same laser power input. Therefore, we determined the laser power curve for the stable die-bonding of each substrate thickness, as shown in Fig. 15. The different substrate thicknesses required for average laser power from thick to thin are 84.0, 69.5, 54.3, 39.8 and 26.1 W. As the substrate is reduced in $0.1 \mathrm{~mm}$ increments, the average laser power decreased by $15 \mathrm{~W}$. Figure 16 shows changes in the thermal stress of carbonized silicon at Point SiC_A as the substrate thickness changed, from which it is noted that thermal stress was affected by temperature as controlled by laser power, but without

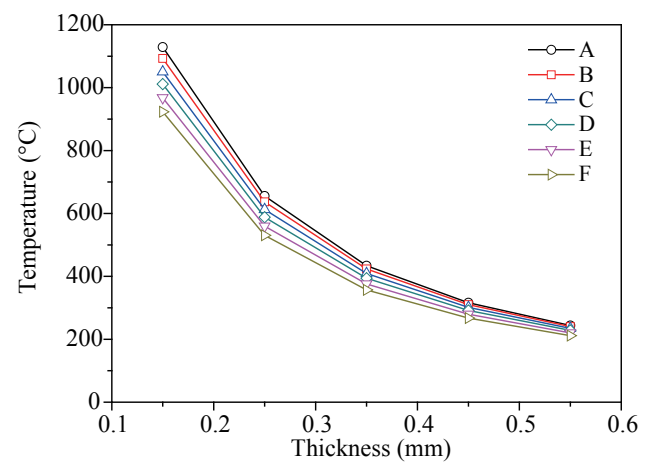

(a)

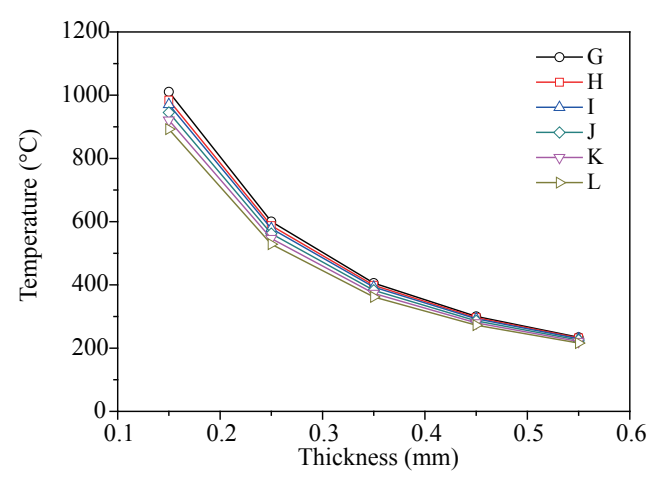

(b)

Fig. 14 (Color online) Changes in temperature at the tin alloy observation point with substrate thickness. (a) A-F and (b) G-L observation points.

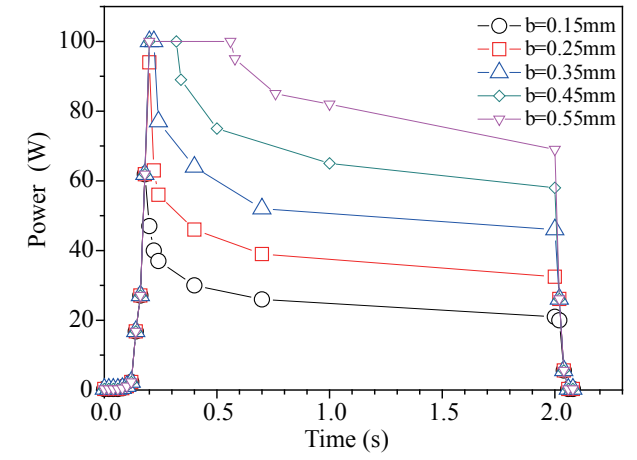

Fig. 15. (Color online) Stable die-bonding laser power for different substrate thicknesses.

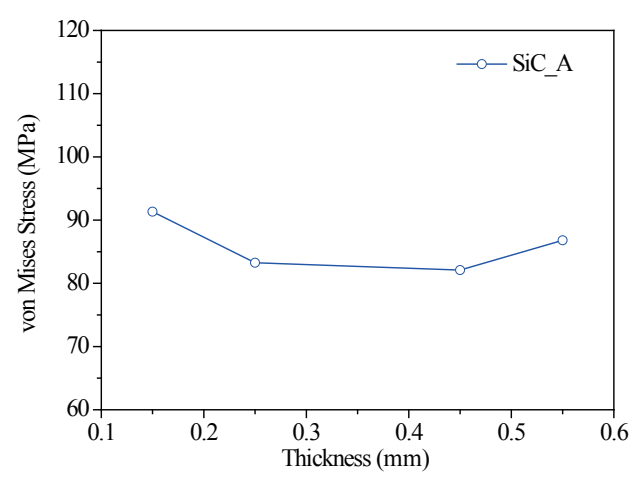

Fig. 16. (Color online) Thermal stress at Point SiC_A as a function of substrate thickness. 
the risk of breaking. Figure 17 shows that the residual stress of the tin alloy changed with substrate thickness, and that the thinner the substrate, the higher the residual stress; residual stress in the 0.15 $\mathrm{mm}$ substrate exceeded by $13 \mathrm{MPa}$ that in the $0.45 \mathrm{~mm}$ substrate. A thicker substrate can certainly provide mechanical protection, but with lower residual stress. However, compared with decreased power from a thin substrate and lower packaging thermal resistance, thinning the substrate as much as possible while maintaining acceptable mechanical properties is suggested.

\subsubsection{Trough depth}

Because each temperature array can be accumulated during a continuous die-bonding process, a trough on the substrate was opened between each array to form temperature fields and avoid this disadvantage. In this section, we discuss the substrate trough's impact on laser die-bonding and the design of five groups of troughs with different depths on an aluminum nitride substrate, ranging within the single array area of a $6.7 \times 6.7 \mathrm{~mm}^{2}$ bonding pad (Figs. 2 and 3), all having a width of 0.3 $\mathrm{mm}$. With respect to etching size, this was a relatively large width, with depths of $0.112,0.168,0.225$, $0.281,0.337$, and $0.393 \mathrm{~mm}$.

Since the temperature of the tin alloy changed significantly with trough depth, we obtained the required laser power curve for each trough depth in the die-bonding, as shown in Fig. 18; the laser powers were $69.5,68.5,67.8,66.4,64.0,61.7$, and $56.4 \mathrm{~W}$. As the trough depth increased every $55 \mathrm{~mm}$ within the shallow trough scope, the average laser power decreased about $1 \mathrm{~W}$, and the deep trough range also changed by 3-5 W. Figure 19 shows that the thermal stress of carbonized silicon changed with trough depth at Point SiC_A, and it can be seen that the thermal stress and temperature changed in the same direction, which indicates that the thermal stress was affected by the quality of the laser power controlled temperature. Figure 20 shows that the residual stress in the tin alloy changed with trough depth, and that the deeper the trough depth, the higher the residual stress in the tin alloy. The residual stress at a depth of $0.393 \mathrm{~mm}$ exceeded by $27 \mathrm{MPa}$ that at a depth of $0.112 \mathrm{~mm}$. Moreover, the residual stresses of carbonized silicon and the epitaxial layer area also increased by $10 \mathrm{MPa}$ as the trough depth increased.

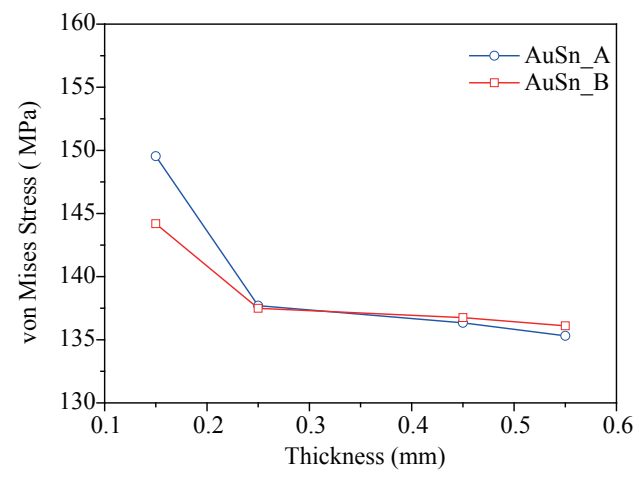

Fig. 17. (Color online) Changes in the residual stress of the tin alloy observation point as a function of substrate thickness.

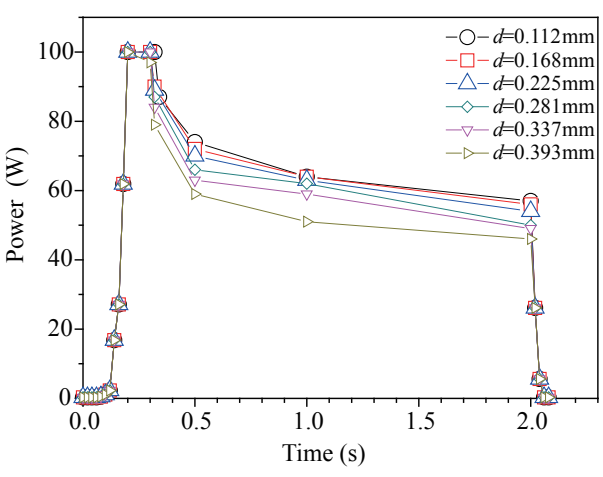

Fig. 18. (Color online) Stable die-bonding laser power at different trough depths. 


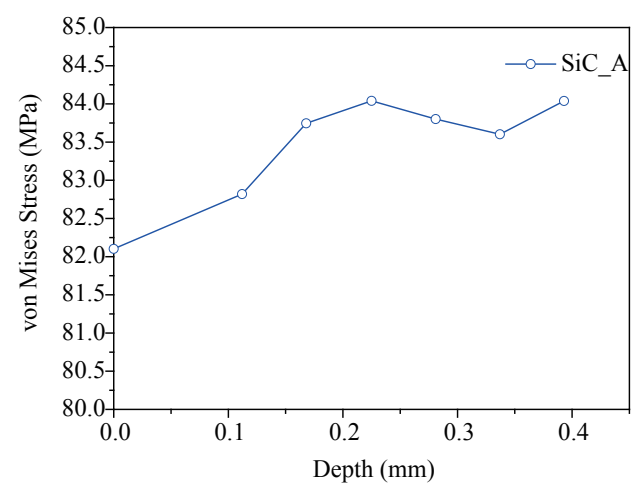

Fig. 19. (Color online) Change in thermal stress at Point SiC_A with trough depth.

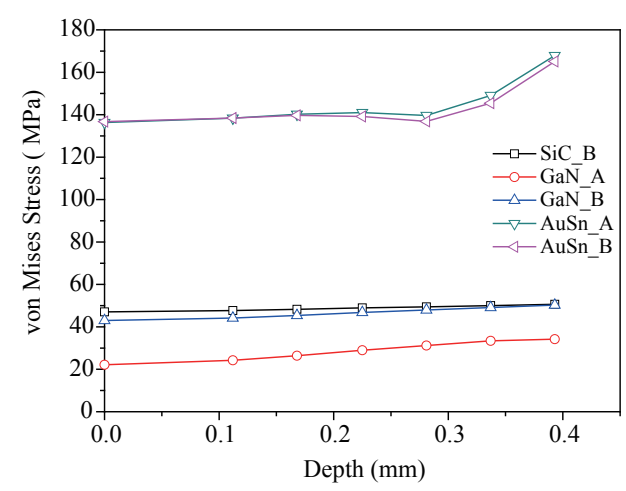

Fig. 20. (Color online) Change in residual stress at each observation point with trough depth.

\section{Conclusions}

Different laser die-bonding process configurations can be evaluated using the thermal-elasticplastic model with the finite element method, and the main contributions of this are as follows.

When tin alloy was heated steadily at $300-325^{\circ} \mathrm{C}$ for $1.8 \mathrm{~s}$, the thermal stress of the carbonized silicon substrate was $82 \mathrm{MPa}$, and there was no risk of breaking. In the laser die-bonding mode, the residual stress of each material on crystal grains was $47 \mathrm{MPa}$ on the carbonized silicon substrate, 43 $\mathrm{MPa}$ on the gallium nitride epitaxial layer, and $136 \mathrm{MPa}$ on tin alloy; this stress is quite unfavorable for product reliability. The results also show that thermal stress and residual stress were affected by process temperature quality. Furthermore, for a single laser, the positive deviation was $0.08 \mathrm{~mm}$, the center of which was determined to be the most uniform location for heating the tin alloy's anode and cathode.

As the substrate was thinned in $0.1 \mathrm{~mm}$ increments, the average power required for the laser decreased by $15 \mathrm{~W}$, but the residual stress in the tin alloy also increased as the substrate become thinner. A trough design in the substrate reduced temperature increased during a continuous diebonding process, and using an array trough with a width of $0.3 \mathrm{~mm}$ that deepened every $55 \mathrm{~mm}$ could save $1.87 \mathrm{~W}$ of laser power. However, the residual stress in the tin alloy was also increased as the trough depth increased.

\section{References}

1 L. Lin, Y. T. Cheng, and K. Najafi: Jpn. J. Appl. Phys. 37 (1998) 1412.

2 Y. T. Cheng, L. Lin, and K. Najafi: J. Microelectromech. Syst. 9 (2000) 3.

3 M. J. Wild, A. Gillner, and R. Poprawe: Sens. Actuators 93 (2001) 63.

4 Y. Tao, A. P. Malshe, and W. D. Brown: Microelectron. Reliability 44 (2004) 251.

5 Y. Tao, A. P. Malshe, W. D. Brown, D. R. Dereus, and S. Cunningham: IEEE Trans. Adv. Packaging 26 (2003) 283.

6 A. W. Y. Tan and F. E. H. Tayof: Sens. Actuators 120 (2005) 550.

7 A. W. Y. Tan, F. E. H. Tay, and J. Zhang: Sens. Actuators 125 (2006) 573.

8 J. W. R. Teo, F. L. Ng, L. S. K. Goi, Y. F. Sun, Wang, Z. F., X. Q. Shi, J. Wei, and G. Y. Li: Microelectron. Eng. 85 (2008) 512. 
9 W. Pittroff, G. Erbert, G. Beister, F. Bugge, A. Klein, A. Knauer, J. Maege, P. Ressel, J. Sebastian, R. Staske, and G. Traenkle: IEEE Trans. Adv. Packaging 24 (2001) 434.

10 J. W. R. Tew, Z. F. Wang, X. Q. Shi, and G. Y. Li: Electron. Packaging Technol. Conf. (2004) pp. 390-395.

11 J. W. R. Tew, X. Q. Shi, and S. Yuan: Mater. Lett. 58 (2004) 2695.

12 J. W. R. Teo, G. Y. Li, M. S. Ling, Z. F. Wang, and X. Q. Shi: Thin Solid Films 515 (2007) 4340.

13 MSC Software Corporation: MSC MARC Product Documentation Vol. B (Element Library, MSC Software Corporation, 2010). 